\title{
Rotational spectroscopy of isotopic species of methyl mercaptan at millimeter and submillimeter wavelengths: $\mathrm{CH}_{3}{ }^{34} \mathrm{SH}^{\star, \star \star}$
}

\author{
Olena Zakharenko ${ }^{1}$, Frank Lewen ${ }^{1}$, Vadim V. Ilyushin ${ }^{2,3}$, Holger S. P. Müller ${ }^{1}$, Stephan Schlemmer ${ }^{1}$, \\ Eugene A. Alekseev ${ }^{2,3}$, Igor Krapivin ${ }^{2}$, Li-Hong Xu (徐丽红) ${ }^{4}$, Ronald M. Lees ${ }^{4}$, Robin Garrod ${ }^{5}$, \\ Arnaud Belloche ${ }^{6}$, and Karl M. Menten ${ }^{6}$

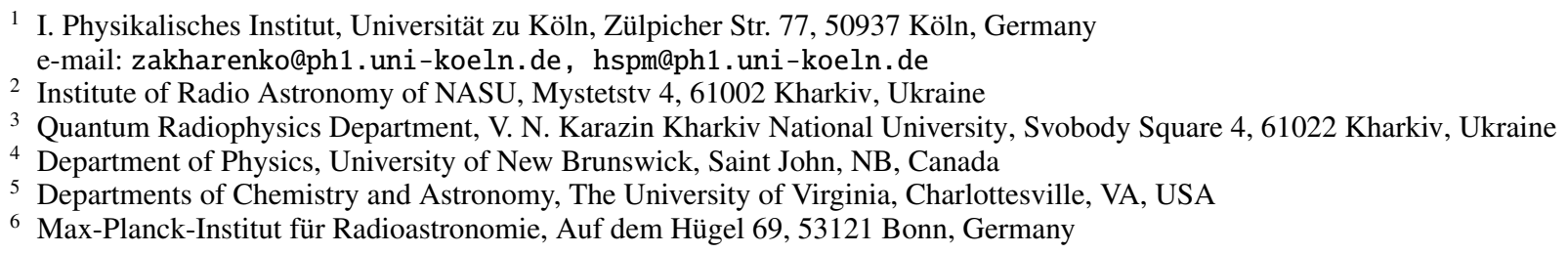

Received 29 March 2019 / Accepted 20 May 2019

\begin{abstract}
Methyl mercaptan $\left(\mathrm{CH}_{3} \mathrm{SH}\right)$ is an important sulfur-bearing species in the interstellar medium, terrestrial environment, and potentially in planetary atmospheres. The aim of the present study is to provide accurate spectroscopic parameters for the most abundant minor isotopolog $\mathrm{CH}_{3}{ }^{34} \mathrm{SH}$ to support radio astronomical observations at millimeter and submillimeter wavelengths. The rotational spectrum of $\mathrm{CH}_{3}{ }^{34} \mathrm{SH}$, which is complicated by the large-amplitude internal rotation of the $\mathrm{CH}_{3}$ group versus the ${ }^{34} \mathrm{SH}$ frame, was investigated in the $49-510 \mathrm{GHz}$ and $1.1-1.5 \mathrm{THz}$ frequency ranges in natural isotopic abundance. The analysis of the spectrum was performed up to the second excited torsional state, and the obtained data were modeled with the RAM36 program. A fit within experimental accuracy was obtained with a RAM Hamiltonian model that uses 72 parameters. Predictions based on this fit are used to search for $\mathrm{CH}_{3}{ }^{34} \mathrm{SH}$ with the Atacama Large Millimeter/submillimeter Array (ALMA) toward the hot molecular core Sgr B2(N2), but blends with emission lines of other species prevent its firm identification in this source.
\end{abstract}

Key words. methods: laboratory: molecular - techniques: spectroscopic - ISM: molecules - astrochemistry - ISM: abundances radio lines: ISM

\section{Introduction}

Sulfur (S) is of importance for astrophysics as one of the constituents of interstellar dust. The studies of the interstellar grains, extracted from the interplanetary dust particles embedded in meteorites, show that sulfur is often included into interstellar silicates (Bradley et al. 1999). Astronomical silicates are one of the fundamental building blocks from which the solar system is assumed to be formed. In addition, sulfur is considered to be one of the tools to study the evolutionary stages of massive stars (Kahane et al. 1988).

The sulfur-bearing molecule methyl mercaptan, $\mathrm{CH}_{3} \mathrm{SH}$, plays an important role in interstellar chemistry and potentially in planetary atmospheres (Vance et al. 2011). It is abundant in some astronomical sources and was first detected in Sgr B2 (Turner 1977). The molecule was observed later toward the high-mass star-forming region G327.3-0.6 (Gibb et al. 2000). Recently, methyl mercaptan was observed in molecular line surveys carried out with the Atacama Millimeter/submillimeter Array (ALMA) towards Sgr B2(N2) (Müller et al. 2016; Belloche et al. 2016)

* The input and output files of the fit are only available at the CDS via anonymous ftp to cdsarc.u-strasbg.fr $(130.79 .128 .5)$ or via http://cdsarc.u-strasbg.fr/viz-bin/qcat?]/A+A/627/ A41

$\star \star$ This manuscript is dedicated to the memory of Li-Hong Xu who passed away at the final stage of writing of the manuscript. at levels that make detection of its ${ }^{34} \mathrm{~S}$ isotopic species promising. The terrestrial abundance of the second-most abundant stable sulfur isotope ${ }^{34} \mathrm{~S}$ is $4.21 \%$. Investigation of the ${ }^{34} \mathrm{~S}$ isotopolog and its detection in the interstellar medium will provide additional information on the ${ }^{32} \mathrm{~S} /{ }^{34} \mathrm{~S}$ abundance ratio, which is of interest for investigation of stellar nucleosynthesis and thus Galactic chemical evolution. With the increased sensitivity and resolution of the ALMA telescope, a large number of new unknown lines is observed compared to the past. A considerable portion of the unknown lines belongs to high rotational or vibrational (including torsional) states of known molecules, as well as to their isotopologs, with $\mathrm{CH}_{3}{ }^{34} \mathrm{SH}$ being a potential contributor.

The rotational spectrum of methyl mercaptan was investigated in the centimeter (Kojima \& Nishikawa 1957; Kojima 1960), millimeter (Bettens et al. 1999; Lees \& Mohammadi 1980; Sastry et al. 1986; Xu et al. 2012), and infrared wavelength regimes (Lees et al. 2018). Recently, the $\mathrm{CH}_{3}{ }^{32} \mathrm{SD}$ isotopolog was studied in the $150-510 \mathrm{GHz}$ frequency range (Zakharenko et al. 2019). This study extended the previous investigation of the $\mathrm{CH}_{3}{ }^{32} \mathrm{SD}$ spectrum performed by Tsunekawa et al. (1989). Concerning the ${ }^{34} \mathrm{~S}$ isotopolog of methyl mercaptan, only one transition, $1_{01} \leftarrow 0_{00}$, was observed (Solimene \& Dailey 1955). One additional series of the ${ }^{34} \mathrm{~S}$ isotopic species of the molecule was found around $31 \mathrm{GHz}$ by Kojima (1960), but assignments were not discussed. We report a global modeling of the rotational transitions of $\mathrm{CH}_{3}{ }^{34} \mathrm{SH}$ in the ground, the first, and 


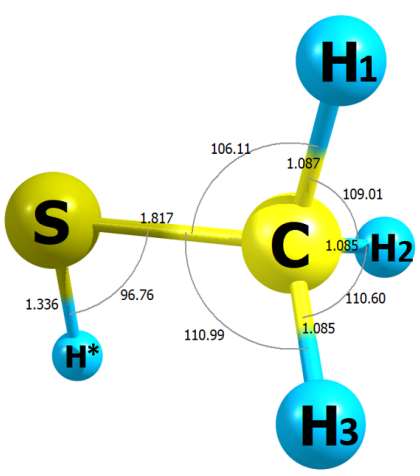

Fig. 1. Schematic view of ${ }^{34} \mathrm{~S}$ methyl mercaptan and equilibrium geometry calculated at MP2/aug-cc-pVTZ level of theory. The structural parameters are shown in angstroms and degrees.

the second excited torsional states. The spectrum is complicated by the large-amplitude internal rotation of the methyl group $\mathrm{CH}_{3}$ with respect to the ${ }^{34} \mathrm{SH}$ frame. Quantum chemical calculations were performed to provide initial estimates of the rotational constants and determine the geometry of $\mathrm{CH}_{3}{ }^{34} \mathrm{SH}$. The aim of the present study is to obtain accurate spectroscopic parameters of the ${ }^{34} \mathrm{~S}$ isotopolog of methyl mercaptan to support astronomical observations by radio telescope arrays, in particular at millimeter and submillimeter wavelengths. The remainder of the manuscript is laid out as follows. Sections 2 and 3 provide details on our quantum chemical calculations and on our laboratory measurements, respectively. The laboratory spectroscopic analysis and discussion are given in Sect. 4. Sections 5 and 6 describe our astronomical observations and the results of our search for $\mathrm{CH}_{3}{ }^{34} \mathrm{SH}$, respectively, while Sect. 7 details the conclusions of our investigations.

\section{Quantum chemical calculations}

All calculations were performed using the Gaussian 09 (G09), Revision A.03, software package (Frisch et al. 2016). The molecular parameters and the harmonic force field of methyl mercaptan were evaluated using the Møller-Plesset second-order theory (MP2; Møller \& Plesset 1934) with aug-cc-pVTZ basis set. The structural parameters were obtained in order to provide an accurate equilibrium structure. The schematic view and the main structural parameters are shown in Fig. 1.

\section{Experimental details}

Measurements in Cologne were done in the frequency ranges of 155-510 GHz and 1.1-1.5 THz using the Cologne $\mathrm{mm} / \mathrm{submm}$ wave and THz spectrometers. An Agilent E8257D synthesizer, referenced to a rubidium standard, together with VDI (Virginia Diodes, Inc.) Amplified Multiplier Chains were employed as frequency sources. The $\mathrm{mm} / \mathrm{submm}$ output radiation was guided through the $5 \mathrm{~m}$ double-pass glass cell (the $\mathrm{THz}$ radiation to the $7 \mathrm{~m}$ glass cell) of $10 \mathrm{~cm}$ in diameter and then to the detectors. We used Schottky diode detectors to detect output frequencies in the $155-510 \mathrm{GHz}$ frequency region and a helium-cooled bolometer (QMC Instruments Ltd.) in the $1.1-1.5 \mathrm{THz}$ frequency region. The measurements were carried out at room temperature and at a pressure of 20-40 $\mu$ bar. The input frequency was modulated at $47.8 \mathrm{kHz}$ in the $155-510 \mathrm{GHz}$ range and at $16.7 \mathrm{kHz}$ in the terahertz range. The modulation amplitude and frequency steps were adjusted to optimize the signal-to-noise ratio $(\mathrm{S} / \mathrm{N})$. The output signal from the detector was detected by a lock-in amplifier in $2 f$ mode to give second-derivative spectra, with a time constant of 20 or $50 \mathrm{~ms}$. Detailed descriptions of the spectrometers may be found in Bossa et al. (2014) and Xu et al. (2012). Methyl mercaptan (purity $\geq 98.0 \%$ ) was purchased from Sigma Aldrich and was used without further purification. The ${ }^{34} \mathrm{~S}$ isotopic species of $\mathrm{CH}_{3} \mathrm{SH}$ was measured in natural abundance.

Measurements in Kharkiv were done in the frequency range of $49-150 \mathrm{GHz}$ using the automated spectrometer of the Institute of Radio Astronomy of NASU (Alekseev et al. 2012). The synthesis of the frequencies in the millimeter wave range was carried out by a two-step frequency multiplication of a reference synthesizer in two phase-lock-loop (PLL) stages. The reference synthesizer is a computer-controlled direct digital synthesizer (DDS AD9851), whose output is upconverted into the $385-430 \mathrm{MHz}$ frequency range. At the first multiplication stage a klystron operating in the $3.4-5.2 \mathrm{GHz}$ frequency range with a narrowband $(1 \mathrm{kHz})$ PLL system was used. At the second multiplication stage, an Istok backward wave oscillator (BWO) was locked to a harmonic of the klystron. A set of BWOs was used to cover the frequency range from 49 to $149 \mathrm{GHz}$. The input frequency was modulated at $11.16 \mathrm{kHz}$ and the output signal from the detector was detected by a lock-in amplifier in $1 f$ mode to give first-derivative spectra. The measurements were carried out at room temperature and at a pressure of 10-20 $\mu \mathrm{bar}$. The uncertainty of the measurements was estimated to be $10 \mathrm{kHz}$ for a relatively strong isolated line $(S / N>10), 30 \mathrm{kHz}$ for weak lines $(2<S / N<10)$, and $100 \mathrm{kHz}$ for very weak lines $(S / N<2)$. Methyl mercaptan was synthesized from a $21 \%$ water solution of sodium thiomethoxide $\mathrm{CH}_{3} \mathrm{SNa}$ (purchased from Sigma Aldrich and used without further purification). The ${ }^{34} \mathrm{~S}$ isotopic species of $\mathrm{CH}_{3} \mathrm{SH}$ was measured in natural abundance.

\section{Laboratory spectroscopic analysis and discussion}

Methyl mercaptan has two dipole moment components, $\mu_{a}=1.312 \mathrm{D}$ and $\mu_{b}=0.758 \mathrm{D}$ (Tsunekawa et al. 1989), thus, both $a$-type and $b$-type transitions are observed. The $a$ axis lies almost parallel to the $\mathrm{S}-\mathrm{C}$ bond at an angle of $1.67^{\circ}$, and the $\mathrm{H}^{*}-\mathrm{S}-\mathrm{C}-\mathrm{H}_{2}$ chain lies in the $a b$ plane with the $\mathrm{H}^{*}-\mathrm{S}$ bond at an angle of $8.43^{\circ}$ to the $b$ axis, see Fig. 1. The $\rho$ axis method (RAM; Kirtman 1962; Lees \& Baker 1968; Hougen et al. 1994), implemented in the RAM36 code (Ilyushin et al. 2010), was used to perform the analyses of the high resolution millimeter-wave and terahertz spectra of methyl mercaptan. The initial predictions were based on a set of the rotational constants obtained from quantum chemical calculations. With the assumption that the ${ }^{34} \mathrm{~S}$ isotopic substitution does not considerably alter the molecular structure, the values of the quartic centrifugal distortion parameters and main internal rotation parameters (such as the internal rotation barrier $V_{3}$, the coupling parameter $\rho$, and the internal rotation constant $F$ ) were fixed at the corresponding values of the $\mathrm{CH}_{3}{ }^{32} \mathrm{SH}$ parent molecule (Xu et al. 2012).

We started our analysis from the search of the series of intense $a$-type $R$-branch transitions with low $K_{a}$ quantum numbers. The part of the spectrum of methyl mercaptan illustrated in Fig. 2 shows the relative intensities of the series of ${ }^{a} R 6$ rotational transitions of $\mathrm{CH}_{3}{ }^{34} \mathrm{SH}$ compared to the main isotopolog. The predicted lines were not in the immediate vicinity of the observed lines. Nevertheless, the assignments of the ground state lines of $\mathrm{CH}_{3}{ }^{34} \mathrm{SH}$ were made due to the similarity in the spectral patterns of the ${ }^{34} \mathrm{~S}$ and the main isotopic species. The assigned transitions were fit, and further analyses proceeded by 


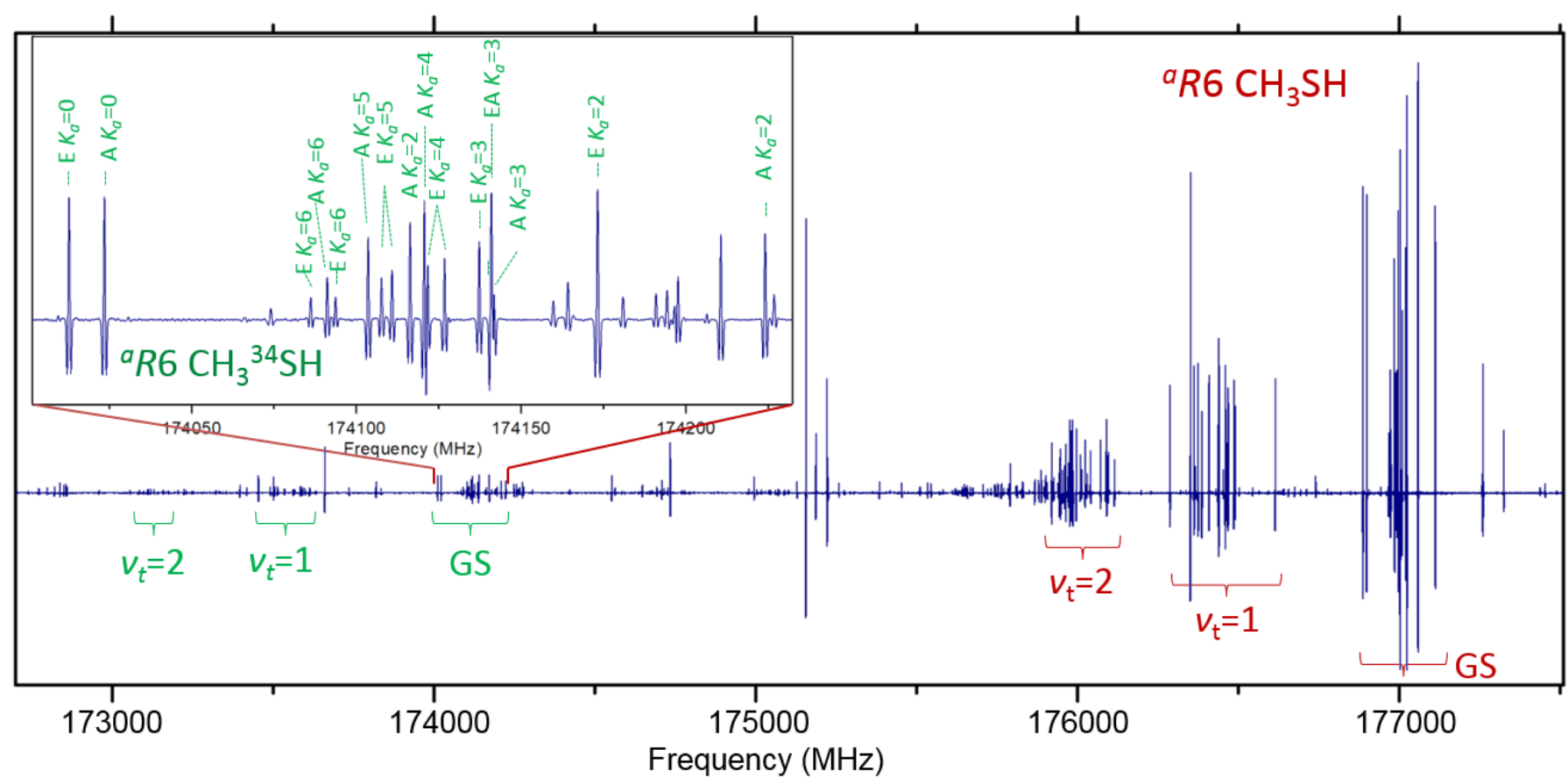

Fig. 2. Partial methyl mercaptan spectrum around $175 \mathrm{GHz}$. The ${ }^{a} R 6$ rotational transitions in the ground as well as the first two excited torsional states of $\mathrm{CH}_{3}{ }^{32} \mathrm{SH}$ are shown on the right and those of $\mathrm{CH}_{3}{ }^{34} \mathrm{SH}$ on the left. The inset contains symmetry (A or E) and $K_{a}$ labels for lines of the ground state of $\mathrm{CH}_{3}{ }^{34} \mathrm{SH}$.

increasing the $J$ and $K_{a}$ quantum numbers. The process of assignment comprises gradual adding of newly measured transitions to the dataset and numerous cycles of improving of the parameter set. A large number of $Q-, P$-, and $R$-branch $b$-type transitions were assigned subsequently, which provided a large dataset for the detailed modeling of the rotational spectrum.

The analyses of the first and second excited states of the methyl torsion were carried out in a similar manner. The predictions for the excited torsional states were calculated based on the set of the ground state parameters of $\mathrm{CH}_{3}{ }^{34} \mathrm{SH}$. Finally, a number of measured transitions in the frequency range $1.1-1.5 \mathrm{THz}$ were added to the dataset. These data did not pose any problem for the fitting process. Figure 3 shows an example of the agreement achieved between predicted and experimental spectra for methyl mercaptan in the $\mathrm{THz}$ region, with the three $Q$-branch series again illustrating the relative intensity ratio between the main and ${ }^{34} \mathrm{~S}$ isotopic species.

In total, 3469 rotational transitions were measured for the ground, the first, and the second excited torsional states of $\mathrm{CH}_{3}{ }^{34} \mathrm{SH}$, which correspond to 3069 fit line frequencies due to blending. Some statistical information on the final fit is presented in Table 1. The estimated uncertainties for measured line frequencies are in the range from 10 to $100 \mathrm{kHz}$ depending on the frequency range and the observed $\mathrm{S} / \mathrm{N}$. The upper limits of the $J$ and $K_{a}$ quantum numbers naturally decrease with energy progression from the ground, over the first, to the second excited torsional states. The full dataset was fit using 72 parameters with an overall weighted root mean square (rms) deviation of 0.83. The input and output files of the final fit are available on the Cologne Database for Molecular Spectroscopy (CDMS; Endres et al. 2016). The 72 parameters from the global fit of the $\mathrm{CH}_{3}{ }^{34} \mathrm{SH}$ spectrum are listed in Table A.1. It should be noted that the final fit converged properly: the relative change in the weighted rms deviation of the fit at the last iteration was about $10^{-7}$; the corrections to the parameter values generated at the last iteration are less than $10^{-4}$ of the calculated parameter confidence intervals; the changes generated at the last iteration in the calculated frequencies are less than $1 \mathrm{kHz}$. Nevertheless, the analysis of the singular value decomposition reveals a group of highly correlated parameters, namely $\rho_{m K}, \rho_{K K}, \rho_{m m}, F_{m m}, F_{m K}$, $F_{K K}, \rho_{m}, F_{K}$. Unfortunately, elimination of any of these correlated parameters leads to a significant worsening of the overall weighted rms deviation of the fit. The problem with the correlations listed above may be caused by small rotation-torsionvibration interactions in the spectra, such as those discussed in $\mathrm{Xu}$ et al. (2012) in connection with the analysis of the main isotopolog of methyl mercaptan. Similar perturbations occur in the spectrum of the ${ }^{34} \mathrm{~S}$ isotopolog and increased rms deviations for the $E$ type, $v_{t}=2$ group of transitions in comparison with other groups is a manifestation of such perturbations. The perturbations from the low-lying, small amplitude vibrations propagating through torsion-rotation interaction down to the low-lying torsional states require inclusion of additional terms that lead to increased correlations among parameters. An additional manifestation of such perturbations in the parameter set is the same order of magnitude of the $V_{6}$ and $V_{9}$ terms in the expansion of the torsional potential function.

We decided to compare our current results with the parameters of the main isotopolog (Xu et al. 2012) and of the deuterated methyl mercaptan (Zakharenko et al. 2019) in order to have a more detailed picture of what is happening with the Hamiltonian model of $\mathrm{CH}_{3} \mathrm{SH}$ upon isotopic substitution. All three fits employ slightly different sets of high-order torsionrotational parameters, in particular due to differences in the datasets. Therefore, we limit ourselves to the comparison of parameters of only up to the fourth order in Table A.2. Compared to the H/D substitution, the ${ }^{34} \mathrm{~S}$ isotopic substitution does not lead to large changes either in the rotational constants or in the main internal rotational parameters $V_{3}, \rho$, and $F$ (the differences for the latter are about 0.1 percent and less). Furthermore, 


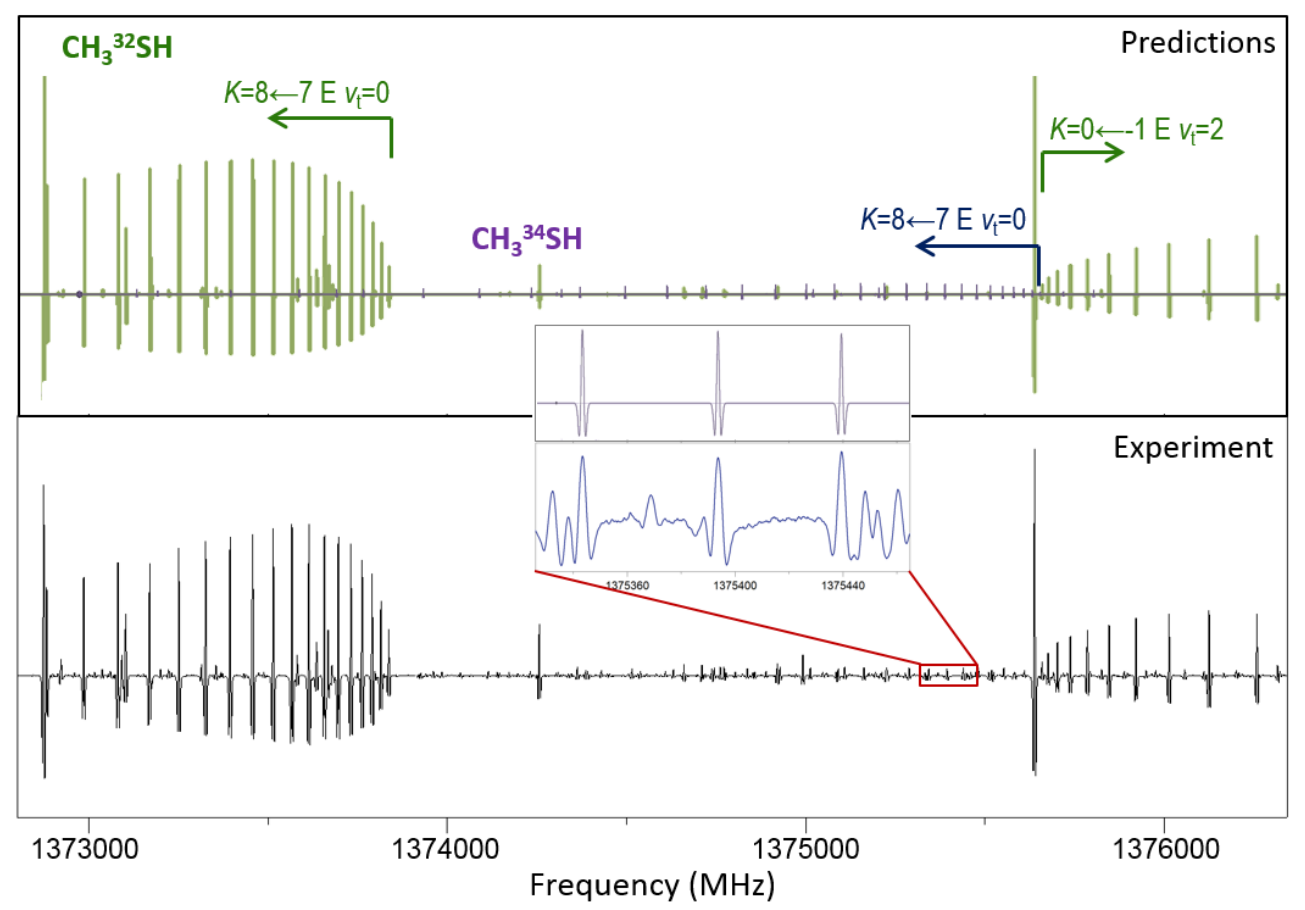

Fig. 3. Partial methyl mercaptan spectrum around $1375 \mathrm{GHz}$. Series of $Q$ branch rotational transitions in the $\mathrm{THz}$ frequency region are shown $(K=8 \leftarrow$ $7, E, v_{t}=0$ and $K=0 \leftarrow-1, E, v_{t}=2$ of $\mathrm{CH}_{3}{ }^{32} \mathrm{SH}$, and $\mathrm{K}=8 \leftarrow 7, E, v_{t}=0$ of $\mathrm{CH}_{3}{ }^{34} \mathrm{SH}$ ).
Table 1. Total number of transitions and other statistical information for the $\mathrm{CH}_{3}{ }^{34} \mathrm{SH}$ data set.

\begin{tabular}{lcccc}
\hline \hline$v_{t}, \operatorname{sym}^{(a)}$ & $N^{(b)}$ & $K_{a, \max }{ }^{(c)}$ & $J_{\max }{ }^{(d)}$ & $\mathrm{rms}^{(e)}$ \\
\hline $0, \mathrm{~A}$ & 993 & 16 & 55 & 41.0 \\
0, E & 918 & 15 & 55 & 39.5 \\
1, A & 504 & 13 & 45 & 39.1 \\
1, E & 501 & 12 & 47 & 27.9 \\
2, A & 235 & 7 & 38 & 32.2 \\
2, E & 318 & 8 & 38 & 63.6 \\
\hline
\end{tabular}

Notes. ${ }^{(a)}$ Torsional level and symmetry label (A or E) of lower and upper states of rotational transition. ${ }^{(b)}$ Number of rotational transitions in a given category. ${ }^{(c)}$ Maximum value of $K_{a}$ quantum number in a given category. ${ }^{(d)}$ Maximum value of $J$ quantum number in a given category. ${ }^{(e)}$ Root mean square (rms) deviation for corresponding group of data in $\mathrm{kHz}$.

many torsion-rotation distortion parameters of the fourth order agree between the ${ }^{34} \mathrm{~S}$ and ${ }^{32} \mathrm{~S}$ isotopologs.

Nevertheless, we observe changes in sign for two parameters, namely $\Delta_{J K}$ and $D_{a b J}$, for the ${ }^{34} \mathrm{~S}$ isotopolog compared to the ${ }^{32} \mathrm{~S}$ isotopolog. At the same time, the sign of the $\Delta_{J K}$ parameter in the ${ }^{34} \mathrm{~S}$ isotopolog coincides with the sign of the corresponding parameter in the SD isotopolog. Although these discrepancies may be caused by the difference in the datasets, it is also possible that the agreement in the sign of the $\Delta_{J K}$ parameter between the ${ }^{34} \mathrm{SH}$ and ${ }^{32} \mathrm{SD}$ isotopologs speaks in favor of additional correlation issues in the $\mathrm{CH}_{3}{ }^{32} \mathrm{SH}$ parameter set, where the number of fourth order parameters exceeds by two the number of allowed parameters for this order, as predicted by the reduction scheme proposed by Nakagawa et al. (1987).

\section{Observations}

We use the Exploring Molecular Complexity with ALMA (EMoCA) spectral line survey performed toward the high-mass star-forming region Sagittarius B2(N) with ALMA to search for the ${ }^{34} \mathrm{~S}$ isotopolog of methyl mercaptan. The observations, data reduction, and method used to identify the detected lines and derive column densities were described in detail in Belloche et al. (2016). In short, the survey has a median angular resolution of $1.6^{\prime \prime}$. It was done with five frequency tunings that fully cover the frequency range from $84.1 \mathrm{GHz}$ to $114.4 \mathrm{GHz}$ with a spectral resolution of $488.3 \mathrm{kHz}\left(1.7\right.$ to $\left.1.3 \mathrm{~km} \mathrm{~s}^{-1}\right)$. The phase center was set at $(\alpha, \delta)_{\mathrm{J} 2000}=\left(17^{\mathrm{h}} 47^{\mathrm{m}} 19.87,-28^{\circ} 22^{\prime} 16^{\prime \prime}(0)\right.$. We focus on the peak position of the hot molecular core Sgr B2(N2) at $(\alpha, \delta)_{\mathrm{J} 2000}=\left(17^{\mathrm{h}} 47^{\mathrm{m}} 19.86,-28^{\circ} 22^{\prime} 13^{\prime \prime}{ }^{\prime} 4\right)$ in the present work.

\section{Astronomical results}

In order to search for rotational lines of the ${ }^{34} \mathrm{~S}$ isotopolog of methyl mercaptan in the EMoCA spectrum of Sgr B2(N2), we started from the best-fit radiative-transfer model we obtained for the ${ }^{32} \mathrm{~S}$ isotopolog for this source (Müller et al. 2016). We assumed the same size of the emitting region $\left(1.4^{\prime \prime}\right)$, temperature $(180 \mathrm{~K})$, linewidth $\left(5.4 \mathrm{~km} \mathrm{~s}^{-1}\right)$, and centroid velocity $\left(73.5 \mathrm{~km} \mathrm{~s}^{-1}\right)$ as those derived for the main isotopolog. We followed Wilson \& Rood (1994) and assumed a ${ }^{32} \mathrm{~S} /{ }^{34} \mathrm{~S}$ isotopic ratio of 22.5, equal to the Solar System value. Given the column density of $3.4 \times 10^{17} \mathrm{~cm}^{-2}$ obtained for the main isotopolog, this implies a column density of $1.5 \times 10^{16} \mathrm{~cm}^{-2}$ for the ${ }^{34} \mathrm{~S}$ isotopolog. With these parameters, we computed a synthetic spectrum of $\mathrm{CH}_{3}{ }^{34} \mathrm{SH}$ under the local thermodynamic equilibrium approximation using Weeds (Maret et al. 2011) and compare it to the observed EMoCA spectrum in Fig. 4.

The synthetic spectrum of $\mathrm{CH}_{3}{ }^{34} \mathrm{SH}$ is consistent with the spectrum of Sgr B2(N2), but most of the strongest transitions expected for $\mathrm{CH}_{3}{ }^{34} \mathrm{SH}$ are, unfortunately, blended with emission from other molecules, which prevents their robust assignment to $\mathrm{CH}_{3}{ }^{34} \mathrm{SH}$. Therefore, the molecule is not securely identified in the EMoCA spectrum. Still, the agreement between the observed and synthetic spectra for the line at $99519 \mathrm{MHz}$ (which is contaminated by other species, though only in its wings) suggests that the molecule is present at the level expected for the ${ }^{32} \mathrm{~S} /{ }^{34} \mathrm{~S}$ isotopic ratio assumed above. 


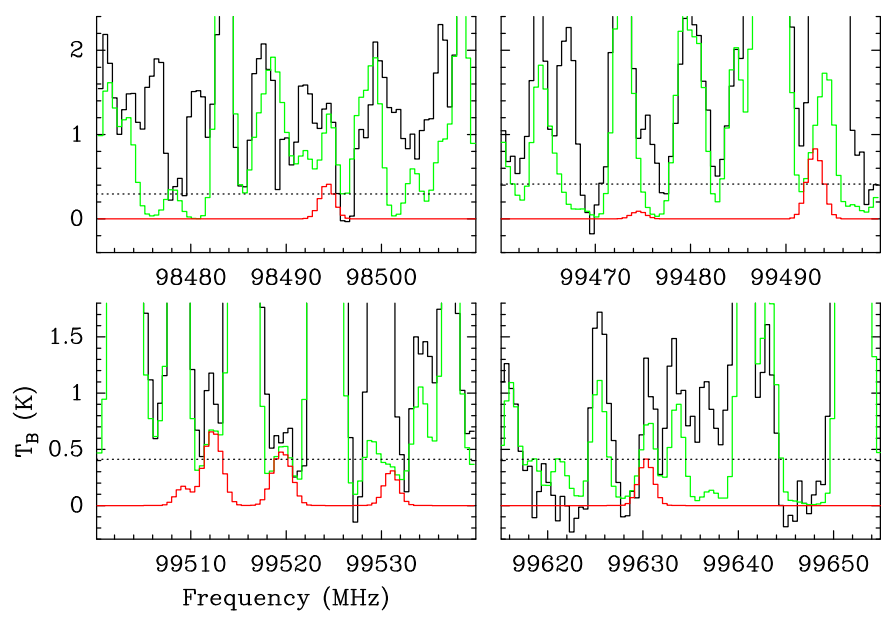

Fig. 4. EMoCA survey detail. This figure shows transitions of $\mathrm{CH}_{3}{ }^{34} \mathrm{SH}$ that are covered by the survey and that contribute significantly to the detected signal toward Sgr B2(N2). The local thermodynamic equilibrium synthetic spectrum of $\mathrm{CH}_{3}{ }^{34} \mathrm{SH}$ is displayed in red and overlaid on the observed spectrum shown in black. The green synthetic spectrum contains the contributions of all molecules identified in our survey so far, including the species shown in red. The y-axis is labeled in brightness temperature units. The dotted line indicates the $3 \sigma$ noise level.

\section{Conclusions}

A new study of the rotational spectrum of the ${ }^{34} \mathrm{~S}$ isotopolog of $\mathrm{CH}_{3} \mathrm{SH}$ was carried out in the frequency ranges of $49-510 \mathrm{GHz}$ and $1.1-1.5 \mathrm{THz}$ in order to provide accurate predictions for astronomical searches. The rotational transitions of the ground, the first, and the second excited torsional states were assigned up to high $J$ and $K_{a}$ quantum numbers (see Table 1) and fit using a RAM Hamiltonian within experimental accuracy. The overall weighted rms deviation of the global fit of 3469 rotational transitions of the $\mathrm{CH}_{3}{ }^{34} \mathrm{SH}$ is 0.83 , indicating that we have an appropriate set of parameters to provide reliable predictions to support astronomical observations. $\mathrm{CH}_{3}{ }^{34} \mathrm{SH}$ was searched for with ALMA between $84 \mathrm{GHz}$ and $114 \mathrm{GHz}$ toward the hot molecular core Sgr B2(N2), but blends with emission lines of other species prevent its firm identification in this frequency range for this source. Nevertheless, the satisfactory agreement between observations and the astronomical model for the least contaminated $\mathrm{CH}_{3}{ }^{34} \mathrm{SH}$ line at $99519 \mathrm{MHz}$ may make a secure detection possible in future observations of this source in a wider frequency range.

A calculated line list of this isotopic species, including information on intensities and calculated uncertainties, will be provided in the catalog section ${ }^{1}$ of the Cologne Database for Molecular Spectroscopy, CDMS (Endres et al. 2016). Files con- taining the experimental lines or parameters along with auxiliary files are available in the data section of the $\mathrm{CDMS}^{2}$.

Acknowledgements. The present study was supported by the Deutsche Forschungsgemeinschaft (DFG) in the framework of the collaborative research grant SFB 956 (project ID 184018867), sub-project B3. O.Z. is funded by the DFG via the Gerätezentrum "Cologne Center for Terahertz Spectroscopy" (project ID SCHL 341/15-1). The research in Kharkiv was carried out under support of the Volkswagen foundation. The assistance of Science and Technology Center in Ukraine is acknowledged (STCU partner project P686). L.H.X. and R.M.L. received support from the Natural Sciences and Engineering Research Council of Canada.

\section{References}

Alekseev, E. A., Motiyenko, R. A., \& Margules, L. 2012, Radio Phys. Radio Astron., 3, 75

Belloche, A., Müller, H. S. P., Garrod, R. T., \& Menten, K. M. 2016, A\&A, 587, A91

Bettens, F. L., Sastry, K. V. L. N., Herbst, E., et al. 1999, ApJ, 510, 789

Bossa, J.-B., Ordu, M. H., Müller, H. S. P., Lewen, F., \& Schlemmer, S. 2014, A\&A, 570, A12

Bradley, J. P., Keller, L. P., Snow, T. P., et al. 1999, Science, 285, 1716

Endres, C. P., Schlemmer, S., Schilke, P., Stutzki, J., \& Müller, H. S. P. 2016, J. Mol. Spectr., 327, 95

Frisch, M. J., Trucks, G. W., \& Schlegel, H. B. 2016, Gaussian 16 Revision A.03 (Wallingford, CT, USA: Gaussian Inc.)

Gibb, E., Nummelin, A., Irvine, W. M., Whittet, D. C. B., \& Bergman, P. 2000, ApJ, 545, 309

Hougen, J., Kleiner, I., \& Godefroid, M. 1994, J. Mol. Spectr., 163, 559

Ilyushin, V. V., Kisiel, Z., Pszczółkowski, L., Mäder, H., \& Hougen, J. T. 2010, J. Mol. Spectr., 259, 26

Kahane, C., Gomez-Conzales, J., Cernicharo, J., \& Guelin, M. 1988, A\&A, 190, 167

Kirtman, B. 1962, J. Chem. Phys., 37, 2516

Kojima, T. 1960, J. Phys. Soc. Jpn., 15, 1284

Kojima, T., \& Nishikawa, T. 1957, J. Phys. Soc. Jpn., 12, 680

Lees, R. M., \& Baker, J. G. 1968, J. Chem. Phys., 48, 5299

Lees, R. M., \& Mohammadi, M. A. 1980, Can. J. Phys., 58, 1640

Lees, R. M., Xu, L.-H., Guislain, B. G., et al. 2018, J. Mol. Spectr., 343, 18

Maret, S., Hily-Blant, P., Pety, J., Bardeau, S., \& Reynier, E. 2011, A\&A, 526, A47

Møller, C., \& Plesset, M. S. 1934, Phys. Rev., 46, 618

Müller, H. S. P., Belloche, A., Xu, L.-H., et al. 2016, A\&A, 587, A92

Nakagawa, K., Tsunekawa, S., \& Kojima, T. 1987, J. Mol. Spectr., 126, 329

Sastry, K. V. L. N., Herbst, E., Booker, R. A., \& Lucia, F. C. D. 1986, J. Mol. Spectr., 116, 120

Solimene, N., \& Dailey, B. P. 1955, J. Chem. Phys., 23, 124

Tsunekawa, S., Taniguchi, I., Tambo, A., et al. 1989, J. Mol. Spectr., 134, 63

Turner, B. E. 1977, ApJ, 213, L75

Vance, S., Christensen, L. E., Webster, C. R., \& Sung, K. 2011, Planet. Space Sci., 59, 299

Wilson, T. L., \& Rood, R. 1994, ARA\&A, 32, 191

Xu, L.-H., Fisher, J., Lees, R. M., et al. 2008, J. Mol. Spectr., 251, 305

Xu, L.-H., Lees, R. M., Crabbe, G. T., et al. 2012, J. Chem. Phys., 137, 104313

Zakharenko, O., Lewen, F., Ilyushin, V. V., et al. 2019, A\&A, 621, A114
2 https://cdms.astro.uni-koeln.de/classic/ predictions/daten/Methanethiol/ 


\section{Appendix A: Additional tables}

Table A.1. Parameters for $\mathrm{CH}_{3}{ }^{34} \mathrm{SH}$.

\begin{tabular}{|c|c|c|c|}
\hline$n_{\mathrm{tr}}^{(a)}$ & Operator $^{(b)}$ & Par. ${ }^{(c, d)}$ & $\mathrm{CH}_{3}{ }^{34} \mathrm{SH}^{(e)}$ \\
\hline $2_{2,0}$ & $p_{\alpha}^{2}$ & $F$ & $15.018101(11)$ \\
\hline 2,0 & $(1-\cos 3 \alpha)$ & $(1 / 2) V_{3}$ & $220.78189(36)$ \\
\hline 2,1 & $p_{\alpha} P_{a}$ & $\rho$ & $0.651352093(16)$ \\
\hline 20,2 & $P_{a}^{2}$ & $A$ & $3.4254266(18)$ \\
\hline 20,2 & $\begin{array}{l}a \\
P_{b}^{2}\end{array}$ & $B$ & $0.424734752(77)$ \\
\hline 20,2 & $P_{c}^{2}$ & $C$ & $0.406539925(82)$ \\
\hline 20,2 & $(1 / 2)\left\{P_{a}, P_{b}\right\}$ & $2 D_{a b}$ & $-0.0155173(17)$ \\
\hline $4_{4,0}$ & $(1-\cos 6 \alpha)$ & $(1 / 2) V_{6}$ & $-0.28034(20)$ \\
\hline 4,0 & $p_{\alpha}^{4}$ & $F_{m}$ & $-0.11330(13) \times 10^{-2}$ \\
\hline $4_{3,1}$ & $p_{\alpha}^{3} P_{a}$ & $\rho_{m}$ & $-0.35743(34) \times 10^{-2}$ \\
\hline $4_{2,2}$ & $P^{2}(1-\cos 3 \alpha)$ & $V_{3 J}$ & $-0.20311808(74) \times 10^{-2}$ \\
\hline $4_{2,2}^{2,2}$ & $P_{a}^{2}(1-\cos 3 \alpha)$ & $V_{3 K}$ & $0.71456(12) \times 10^{-2}$ \\
\hline $4_{2,2}$ & $\left(P_{b}^{2}-P_{c}^{2}\right)(1-\cos 3 \alpha)$ & $V_{3 b c}$ & $-0.83920(12) \times 10^{-4}$ \\
\hline $4_{2,2}$ & $(1 / 2)\left\{P_{a}, P_{b}\right\}(1-\cos 3 \alpha)$ & $V_{3 a b}$ & $0.1207686(89) \times 10^{-1}$ \\
\hline $4_{2,2}$ & $p_{\alpha}^{2} P^{2}$ & $F_{J}$ & $-0.3000547(70) \times 10^{-4}$ \\
\hline $4_{2,2}$ & $p_{\alpha}^{2} P_{a}^{2}$ & $F_{K}$ & $-0.47975(33) \times 10^{-2}$ \\
\hline $4_{2,2}^{2,2}$ & $(1 / 2)\left\{P_{a}, P_{c}\right\} \sin 3 \alpha$ & $D_{3 a c}$ & $0.144733(33) \times 10^{-1}$ \\
\hline $4_{2,2}^{2,2}$ & $(1 / 2)\left\{P_{b}, P_{c}\right\} \sin 3 \alpha$ & $D_{3 b c}$ & $0.901353(76) \times 10^{-3}$ \\
\hline $4_{1,3}$ & $p_{\alpha} P_{a} P^{2}$ & $\rho_{J}$ & $-0.413515(11) \times 10^{-4}$ \\
\hline $4_{1,3}$ & $p_{\alpha} P_{a}^{3}$ & $\rho_{K}$ & $-0.29544(14) \times 10^{-2}$ \\
\hline $4_{1,3}$ & $(1 / 2)\left\{P_{a},\left(P_{b}^{2}-P_{c}^{2}\right)\right\} p_{\alpha}$ & $\rho_{b c}$ & $-0.19410(77) \times 10^{-4}$ \\
\hline $4_{0,4}$ & $P^{4}$ & $-\Delta_{J}$ & $-0.523063(13) \times 10^{-6}$ \\
\hline $4_{0,4}$ & $P^{2} P_{a}^{2}$ & $-\Delta_{J K}$ & $-0.1737732(50) \times 10^{-4}$ \\
\hline $4_{0,4}$ & $P_{a}^{4}$ & $-\Delta_{K}$ & $-0.69678(23) \times 10^{-3}$ \\
\hline $4_{0,4}$ & $P^{2}\left(P_{b}^{2}-P_{c}^{2}\right)$ & $-2 \delta_{J}$ & $-0.435261(47) \times 10^{-7}$ \\
\hline $4_{0,4}$ & $(1 / 2)\left\{P_{a}^{2},\left(P_{b}^{2}-P_{c}^{2}\right)\right\}$ & $-2 \delta_{K}$ & $-0.19133(74) \times 10^{-4}$ \\
\hline $4_{0,4}^{, 4}$ & $(1 / 2) P^{2}\left\{P_{a}, P_{b}\right\}$ & $D_{a b J}$ & $0.20067(82) \times 10^{-6}$ \\
\hline $6_{6,0}$ & $(1-\cos 9 \alpha)$ & $(1 / 2) V_{9}$ & $0.11644(33)$ \\
\hline $6_{6,0}$ & $p_{\alpha}^{6}$ & $F_{m m}$ & $-0.3210(37) \times 10^{-5}$ \\
\hline $6_{5,1}$ & $p_{\alpha}^{5} P_{a}$ & $\rho_{m m}$ & $-0.1198(15) \times 10^{-4}$ \\
\hline $6_{4,2}$ & $P^{2}(1-\cos 6 \alpha)$ & $V_{6 J}$ & $-0.15588(23) \times 10^{-4}$ \\
\hline $\begin{array}{l}4,2 \\
64,2\end{array}$ & $P_{a}^{2}(1-\cos 6 \alpha)$ & $V_{6 K}$ & $-0.846(28) \times 10^{-4}$ \\
\hline $6_{4,2}$ & $(1 / 2)\left\{P_{a}, P_{b}\right\}(1-\cos 6 \alpha)$ & $V_{6 a b}$ & $0.7833(69) \times 10^{-4}$ \\
\hline $6_{4,2}$ & $\left(P_{b}^{2}-P_{c}^{2}\right)(1-\cos 6 \alpha)$ & $V_{6 b c}$ & $-0.26332(24) \times 10^{-4}$ \\
\hline 64,2 & $(1 / 2)\left\{P_{a}, P_{c}\right\} \sin 6 \alpha$ & $D_{6 a c}$ & $0.2798(22) \times 10^{-3}$ \\
\hline 64,2 & $(1 / 2)\left\{P_{b}, P_{c}\right\} \sin 6 \alpha$ & $D_{6 b c}$ & $0.1747(11) \times 10^{-4}$ \\
\hline 64,2 & $(1 / 2)\left\{P_{b}, P_{c}, p_{\alpha}^{2}, \sin 3 \alpha\right\}$ & $D_{3 b c m}$ & $0.5133(20) \times 10^{-5}$ \\
\hline $6_{4,2}$ & $p_{\alpha}^{4} P^{2}$ & $F_{m J}$ & $0.2179(37) \times 10^{-8}$ \\
\hline 64,2 & $p_{\alpha}^{4} P_{a}^{2}$ & $F_{m K}$ & $-0.1827(24) \times 10^{-4}$ \\
\hline 6,2 & $p_{\alpha}^{3} P_{a}^{a} P^{2}$ & $\rho_{m J}$ & $0.502(10) \times 10^{-8}$ \\
\hline $6_{3,3}$ & $p_{\alpha}^{3} P_{a}^{3}$ & $\rho_{m K}$ & $-0.1448(21) \times 10^{-4}$ \\
\hline $6_{3,3}$ & $(1 / 2)\left\{P_{a}, P_{b}, P_{c}, p_{\alpha}, \sin 3 \alpha\right\}$ & $\rho_{b c 3}$ & $0.3849(14) \times 10^{-5}$ \\
\hline $6_{2,4}$ & $P^{4}(1-\cos 3 \alpha)$ & $V_{3 J J}$ & $0.4541(19) \times 10^{-8}$ \\
\hline $6_{2,4}$ & $P^{2} P_{g}^{2}(1-\cos 3 \alpha)$ & $V_{3 J K}$ & $-0.22294(16) \times 10^{-6}$ \\
\hline $6_{2,4}^{2,4}$ & $P_{\alpha}^{4}(1-\cos 3 \alpha)$ & $V_{3 K K}$ & $0.4741(21) \times 10^{-6}$ \\
\hline $6_{2,4}$ & $(1 / 2) P^{2}\left\{P_{a}, P_{b}\right\}(1-\cos 3 \alpha)$ & $V_{3 a b J}$ & $-0.15652(65) \times 10^{-6}$ \\
\hline $6_{2,4}$ & $P^{2}\left(P_{b}^{2}-P_{c}^{2}\right)(1-\cos 3 \alpha)$ & $V_{3 b c J}$ & $0.7677(48) \times 10^{-9}$ \\
\hline $6_{2,4}$ & $(1 / 2)\left\{P_{b}^{2}, P_{c}^{2}\right\} \cos 3 \alpha$ & $V_{3 b 2 c 2}$ & $0.400(14) \times 10^{-8}$ \\
\hline $6_{2,4}$ & $p_{\alpha}^{2} P^{4}$ & $F_{J J}$ & $0.1207(22) \times 10^{-9}$ \\
\hline $6_{2,4}$ & $p_{\alpha}^{2} P_{a}^{2} P^{2}$ & $F_{J K}$ & $0.614(12) \times 10^{-8}$ \\
\hline $6_{2,4}$ & $p_{\alpha}^{2} P_{a}^{4}$ & $F_{K K}$ & $-0.617(10) \times 10^{-5}$ \\
\hline $6_{2,4}$ & $\begin{array}{l}P_{\alpha^{2}} a \\
(1 / 2)\left\{P_{b}^{2}, P_{c}^{2}\right\} p_{\alpha}^{2}\end{array}$ & $F_{b 2 c 2}$ & $-0.338(17) \times 10^{-9}$ \\
\hline $6_{2,4}$ & $(1 / 2) P^{2}\left\{P_{a}, P_{c}\right\} \sin 3 \alpha$ & $D_{3 a c J}$ & $-0.512(16) \times 10^{-7}$ \\
\hline $6_{2,4}$ & $(1 / 2)\left\{P_{a}^{3}, P_{c}\right\} \sin 3 \alpha$ & $D_{3 a c K}$ & $-0.601(26) \times 10^{-6}$ \\
\hline
\end{tabular}

Notes. ${ }^{(a)} n=t+r$, where $n$ is the total order of the operator, $t$ is the order of the torsional part, and $r$ is the order of the rotational part, respectively. The ordering scheme of Nakagawa et al. (1987) is used. ${ }^{(b)}\{A, B, C, D\}=A B C D+D C B A .\{A, B, C\}=A B C+C B A .\{A, B\}=A B+B A$. The product of the operator in the first column of a given row and the parameter in the third column of that row gives the term currently used in the torsion-rotation Hamiltonian of the program, except for $F, \rho$, and $A_{\mathrm{RAM}}$, which occur in the Hamiltonian in the form of $F\left(p_{a}+\rho P_{a}\right)^{2}+A_{\mathrm{RAM}} P_{a}^{2}$. ${ }^{(c)}$ Parameter nomenclature based on the subscript procedure of Xu et al. (2008). ${ }^{(d)}$ Values of the parameters are in $\mathrm{cm}^{-1}$, except for $\rho$, which is unitless. ${ }^{(e)}$ Statistical uncertainties are given in parentheses as one standard uncertainty in units of the last digits. 
Table A.1. continued.

\begin{tabular}{llll}
\hline \hline$n_{\text {tr }}{ }^{(a)}$ & Operator ${ }^{(b)}$ & Par. ${ }^{(c, d)}$ & $\mathrm{CH}_{3}{ }^{34} \mathrm{SH}^{(e)}$ \\
\hline $6_{2,4}$ & $(1 / 2) P^{2}\left\{P_{b}, P_{c}\right\} \sin 3 \alpha$ & $D_{3 b c J}$ & $-0.12267(38) \times 10^{-7}$ \\
$6_{1,5}$ & $p_{\alpha} P_{a} P^{4}$ & $\rho_{J J}$ & $0.19908(96) \times 10^{-9}$ \\
$6_{1,5}$ & $p_{\alpha} P_{a}^{3} P^{2}$ & $\rho_{J K}$ & $0.4053(64) \times 10^{-8}$ \\
$6_{1,5}$ & $p_{\alpha} P_{a}^{5}$ & $\rho_{K K}$ & $-0.1289(26) \times 10^{-5}$ \\
$6_{1,5}$ & $(1 / 2) P^{2}\left\{P_{a},\left(P_{b}^{2}-P_{c}^{2}\right)\right\} p_{\alpha}$ & $\rho_{b c J}$ & $0.934(23) \times 10^{-10}$ \\
$6_{1,5}$ & $(1 / 2)\left\{P_{a}, P_{b}^{2}, P_{c}^{2}\right\} p_{\alpha}$ & $\rho_{b 2 c 2}$ & $-0.6003(67) \times 10^{-9}$ \\
$6_{0,6}$ & $P^{6}$ & $\Phi_{J}$ & $-0.26953(51) \times 10^{-12}$ \\
$6_{0,6}$ & $P^{4} P_{a}^{2}$ & $\Phi_{J K}$ & $0.6360(20) \times 10^{-10}$ \\
$6_{0,6}$ & $P^{2} P_{a}^{4}$ & $\Phi_{K J}$ & $0.1146(14) \times 10^{-8}$ \\
$6_{0,6}$ & $P_{a}^{6}$ & $\Phi_{K}$ & $-0.913(29) \times 10^{-7}$ \\
$6_{0,6}$ & $(1 / 2) P^{2}\left\{P_{a}^{2},\left(P_{b}^{2}-P_{c}^{2}\right)\right\}$ & $2 \phi_{J K}$ & $0.887(21) \times 10^{-10}$ \\
$8_{6,2}$ & $P^{2}(1-\cos 9 \alpha)$ & $V_{9 J}$ & $-0.2513(38) \times 10^{-5}$ \\
$8_{6,2}$ & $P_{a}^{2}(1-\cos 9 \alpha)$ & $V_{9 K}$ & $-0.548(35) \times 10^{-4}$ \\
$8_{6,2}$ & $(1 / 2)\left\{P_{a}, P_{c}\right\} \sin 9 \alpha$ & $D_{9 a c}$ & $0.648(14) \times 10^{-4}$ \\
$8_{4,4}$ & $P^{4}(1-\cos 6 \alpha)$ & $V_{6 J J}$ & $0.1254(42) \times 10^{-8}$ \\
$8_{4,4}$ & $P^{2} P_{a}^{2}(1-\cos 6 \alpha)$ & $V_{6 J K}$ & $-0.2418(23) \times 10^{-7}$ \\
$8_{4,4}$ & $P^{2}\left(P_{b}^{2}-P_{c}^{2}\right)(1-\cos 6 \alpha)$ & $V_{6 b c J}$ & $0.695(12) \times 10^{-9}$ \\
$8_{4,4}$ & $(1 / 2)\left\{P_{b}^{2}, P_{c}^{2}\right\} \cos 6 \alpha$ & $V_{6 b 2 c 2}$ & $0.674(32) \times 10^{-8}$ \\
\hline
\end{tabular}

Table A.2. Comparison of the low-order parameters of $\mathrm{CH}_{3}{ }^{34} \mathrm{SH}, \mathrm{CH}_{3}{ }^{32} \mathrm{SH}$, and $\mathrm{CH}_{3}{ }^{32} \mathrm{SD}$.

\begin{tabular}{|c|c|c|c|c|c|}
\hline$n_{\mathrm{tr}}(a)$ & Operator $^{(b)}$ & Par. ${ }^{(c, d)}$ & $\mathrm{CH}_{3}{ }^{34} \mathrm{SH}^{(e)}$ & $\mathrm{CH}_{3}{ }^{32} \mathrm{SH}^{(e, f)}$ & $\mathrm{CH}_{3}{ }^{32} \mathrm{SD}^{(e, f)}$ \\
\hline $2_{2,0}$ & $p_{\alpha}^{2}$ & $F$ & $15.018101(11)$ & $15.04020465(66)$ & $10.3520639(31)$ \\
\hline 2,0 & $(1 / 2)(1-\cos 3 \alpha)$ & $V_{3}$ & $441.56378(72)$ & $441.442236(10)$ & $435.42500(24)$ \\
\hline 2,1 & $p_{\alpha} P_{a}$ & $\rho$ & $0.651352093(16)$ & $0.651856026(13)$ & $0.493517098(11)$ \\
\hline 20,2 & $P_{a}^{2}$ & $A$ & $3.4254266(18)$ & $3.42808445(84)$ & $2.59513758(20)$ \\
\hline $2,0,2$ & $P_{b}^{a}$ & $B$ & $0.424734752(77)$ & $0.43201954(87)$ & $0.42517153(13)$ \\
\hline 2 & $P_{c}^{2}$ & $C$ & $0.406539925(82)$ & $0.41325076(83)$ & $0.39176839(13)$ \\
\hline 2,2 & $\left\{P_{a}, P_{b}\right\}$ & $D_{a b}$ & $-0.00775864(85)$ & $-0.0073126(59)$ & $0.0053655(12)$ \\
\hline $4_{4,0}$ & $(1 / 2)(1-\cos 6 \alpha)$ & $V_{6}$ & $-0.56068(40)$ & $-0.572786(15)$ & $-0.86918(20)$ \\
\hline 44,0 & $p_{\alpha}^{4}$ & $F_{m}$ & $-0.11330(13) \times 10^{-2}$ & $-0.114016(10) \times 10^{-2}$ & $-0.38535(20) \times 10^{-3}$ \\
\hline $4_{3,1}$ & $p_{\alpha}^{3} P_{a}$ & $\rho_{m}$ & $-0.35743(34) \times 10^{-2}$ & $-0.360009(28) \times 10^{-2}$ & $-0.93969(42) \times 10^{-3}$ \\
\hline $4_{2,2}$ & $P^{2}(1-\cos 3 \alpha)$ & $V_{3 J}$ & $-0.20311808(74) \times 10^{-2}$ & $-0.217540(84) \times 10^{-2}$ & $-0.19405784(64) \times 10^{-2}$ \\
\hline $4_{2,2}$ & $P_{a}^{2}(1-\cos 3 \alpha)$ & $V_{3 K}$ & $0.71456(12) \times 10^{-2}$ & $0.724978(19) \times 10^{-2}$ & $0.685147(15) \times 10^{-2}$ \\
\hline $4_{2,2}$ & $\left(P_{b}^{2}-P_{c}^{2}\right)(1-\cos 3 \alpha)$ & $V_{3 b c}$ & $-0.83920(12) \times 10^{-4}$ & $-0.92104(47) \times 10^{-4}$ & $-0.15679(13) \times 10^{-3}$ \\
\hline $4_{2,2}^{2,2}$ & $\left\{P_{a}^{b}, P_{b}\right\}(1-\cos 3 \alpha)$ & $V_{3 a b}$ & $0.603843(45) \times 10^{-2}$ & $0.61562(30) \times 10^{-2}$ & $0.443895(41) \times 10^{-2}$ \\
\hline $4_{2,2}^{2,2}$ & $p_{\alpha}^{2} P^{2}$ & $F_{J}$ & $-0.3000547(70) \times 10^{-4}$ & $-0.8106(38) \times 10^{-4}$ & $-0.2823067(53) \times 10^{-4}$ \\
\hline $4_{2,2}$ & $p_{\alpha}^{2} P_{a}^{2}$ & $F_{K}$ & $-0.47975(33) \times 10^{-2}$ & $-0.483287(30) \times 10^{-2}$ & $-0.123322(32) \times 10^{-2}$ \\
\hline $4_{2,2}$ & $p_{\alpha}^{2}\left\{P_{a}, P_{b}\right\}$ & $F_{a b}$ & - & $0.843(45) \times 10^{-4}$ & $0.7807(70) \times 10^{-4}$ \\
\hline $4_{2,2}$ & $2 p_{\alpha}^{2}\left(P_{b}^{2}-P_{c}^{2}\right)$ & $F_{b c}$ & - & $0.0536(41) \times 10^{-4}$ & $0.102769(43) \times 10^{-4}$ \\
\hline $4_{2,2}$ & $\left\{P_{a}, P_{c}\right\} \sin 3 \alpha$ & $D_{3 a c}$ & $0.072366(16) \times 10^{-1}$ & $0.1036(15) \times 10^{-1}$ & $0.11336(45) \times 10^{-1}$ \\
\hline $4_{2,2}$ & $\left\{P_{b}, P_{c}\right\} \sin 3 \alpha$ & $D_{3 b c}$ & $0.450676(38) \times 10^{-3}$ & $0.665(14) \times 10^{-3}$ & $0.140496(27) \times 10^{-2}$ \\
\hline $4_{1,3}$ & $p_{\alpha} P_{a} P^{2}$ & $\rho_{J}$ & $-0.413515(11) \times 10^{-4}$ & $-0.4726(54) \times 10^{-4}$ & $-0.369536(28) \times 10^{-4}$ \\
\hline $4_{1,3}$ & $p_{\alpha} P_{a}^{3}$ & $\rho_{K}$ & $-0.29544(14) \times 10^{-2}$ & $-0.30381(74) \times 10^{-2}$ & $-0.75792(11) \times 10^{-3}$ \\
\hline $4_{1,3}$ & $p_{\alpha}\left\{P_{a}^{2}, P_{b}\right\}$ & $\rho_{a b}$ & - & $0.999(67) \times 10^{-4}$ & $0.1017(10) \times 10^{-3}$ \\
\hline $4_{1,3}$ & $p_{\alpha}\left\{P_{a},\left(P_{b}^{2}-P_{c}^{2}\right)\right\}$ & $\rho_{b c}$ & $-0.09705(39) \times 10^{-4}$ & $-0.0462(39) \times 10^{-4}$ & - \\
\hline $4_{0,4}$ & $-P^{4} \quad D \quad-m$ & $\Delta_{J}$ & $0.523063(13) \times 10^{-6}$ & $0.538140(23) \times 10^{-6}$ & $0.4876778(87) \times 10^{-6}$ \\
\hline $4_{0,4}$ & $-P^{2} P_{a}^{2}$ & $\Delta_{J K}$ & $0.1737732(50) \times 10^{-4}$ & $-0.066(26) \times 10^{-5}$ & $0.143777(64) \times 10^{-4}$ \\
\hline $4_{0,4}$ & $-P_{a}^{4}$ & $\Delta_{K}$ & $0.69678(23) \times 10^{-3}$ & $0.7425(48) \times 10^{-3}$ & $0.178662(17) \times 10^{-3}$ \\
\hline $4_{0,4}$ & $-2 P^{2}\left(P_{b}^{2}-P_{c}^{2}\right)$ & $\delta_{J}$ & $0.217630(24) \times 10^{-7}$ & $0.224788(88) \times 10^{-7}$ & $0.384636(10) \times 10^{-7}$ \\
\hline $4_{0,4}$ & $-\left\{P_{a}^{2},\left(P_{b}^{2}-P_{c}^{2}\right)\right\}$ & $\delta_{K}$ & $0.09566(37) \times 10^{-4}$ & $0.10483(32) \times 10^{-4}$ & $0.090811(35) \times 10^{-4}$ \\
\hline $4_{0,4}$ & $P^{2}\left\{P_{a}, P_{b}\right\}$ & $D_{a b J}$ & $0.10033(41) \times 10^{-6}$ & $-0.956(60) \times 10^{-7}$ & - \\
\hline \multirow[t]{2}{*}{$4_{0,4}$} & $\left\{P_{a}^{3}, P_{b}\right\}$ & $D_{a b K}$ & - & $0.202(23) \times 10^{-4}$ & $0.2750(34) \times 10^{-4}$ \\
\hline & & $\theta_{\mathrm{RAM}}$ & $-0.15^{\circ}$ & $-0.14^{\circ}$ & $0.14^{\circ}$ \\
\hline
\end{tabular}

Notes. ${ }^{(a)} n=t+r$, where $n$ is the total order of the operator, $t$ is the order of the torsional part, and $r$ is the order of the rotational part, respectively. The ordering scheme of Nakagawa et al. (1987) is used. ${ }^{(b)}\{A, B, C, D\}=A B C D+D C B A .\{A, B, C\}=A B C+C B A .\{A, B\}=A B+B A$. The product of the operator in the first column of a given row and the parameter in the third column of that row gives the term currently used in the torsion-rotation Hamiltonian of the program, except for $F, \rho$ and $A_{\mathrm{RAM}}$, which occur in the Hamiltonian in the form of $F\left(p_{a}+\rho P_{a}\right)^{2}+A_{\mathrm{RAM}} P_{a}^{2}$. ${ }^{(c)}$ Parameter nomenclature is based on the subscript procedure of Xu et al. (2008). ${ }^{(d)}$ Values of the parameters are in $\mathrm{cm}^{-1}$, except for $\rho$, which is unitless, and except for $\theta_{\text {RAM }}$, which is in degrees. ${ }^{(e)}$ Statistical uncertainties are given in parentheses as one standard uncertainty in units of the last digits. ${ }^{(f)}$ Not all the parameters used for the analysis in Xu et al. (2012) and Zakharenko et al. (2019) listed here. 